
\title{
GYNECOLOGICAL CANCE \\ (6) OPEN ACCESS \\ Evaluation of portable colposcopy and human papillomavirus testing for screening of cervical cancer in rural China
}

Haley Newman, ${ }^{1,2}$ Jilin Hu, ${ }^{3}$ Xiao Li, ${ }^{4}$ Jing He, ${ }^{3,4}$ Leslie Bradford, ${ }^{5}$ Songmei Shan, ${ }^{4}$ Xiaomei Wu, ${ }^{3,4}$ Bin Zhu, ${ }^{6}$ Wenyang Yang, ${ }^{6}$ Bingqin Fu, ${ }^{6}$ Baosheng Zhu, ${ }^{3,4}$ Guangping Gao ${ }^{7}$

For numbered affiliations see end of article.

Correspondence to

Baosheng Zhu, Department of Obstetricsand Gynecology, National Health Commission's Key Laboratory for Healthy Births in WesternChina, First People's Hospital of Yunnan Province, Yunnan 650032 China; bszhu@aliyun.com

$\mathrm{HN}$ and $\mathrm{JH}$ contributed equally.

Received 21 August 2018 Revised 29 September 2018 Accepted 8 October 2018
Check for updates

(C) IGCS and ESGO 2019. Re-use permitted under CC BY-NC. No commercial re-use. Published by BMJ.

To cite: Newman H, Hu J, Li X, et al. Int J Gynecol Cancer 2019;29:23-27.

HIGHLIGHTS

- Portable colposcopy is an effective and easy tool for cervical cancer screening in remote, low-resource settings.

- Human papillomavirus testing with portable colposcopy has higher sensitivity for detection than either method alone.

- Portable colposcopy should be considered part of the screening program in low-resource settings in China.

\section{ABSTRACT}

Objective To evaluate the use of a portable, rechargeable colposcope combined with human papillomavirus (HPV) testing, as compared with HPV testing alone, for screening of cervical cancer and pre-cancerous lesions.

Methods This was a cross-sectional study among 488 women in Baoshan County, Yunnan. The women underwent HPV testing followed by Gynocular portable colposcopy with visual inspection with acetic acid. Obvious lesions were biopsied. If portable colposcopy testing was negative but HPV testing was positive, the women underwent follow-up testing with thin-prep cytology and traditional colposcopy. Cervical biopsies were performed for any abnormalities. Histopathology was followed up with diagnosis and treatment.

Results Among 488 women screened with portable colposcopy, 24 women underwent biopsy based on positive colposcopy screening. Of these 24 women, three were HPV positive and 21 were HPV negative. Five women had cervical intra-epithelial neoplasia (CIN) I and one had advanced cervical cancer. Forty-six women tested positive for HPV. Three of these women had screened positive on preliminary colposcopy, with one positive for CIN III/squamous cell carcinoma and one woman with CIN I. Forty-three women underwent follow-up testing with thin-prep cytology. Two women had atypical squamous cells of undetermined significance and five had low-grade squamous intra-epithelial lesions and were biopsied; three women had CIN I, one had CIN II and one had CIN III. HPV testing and portable colposcopy was more sensitive but slightly less specific than portable colposcopy or HPV testing alone.

Conclusion While HPV testing has high sensitivity and specificity for the detection of pre-cancerous and cancerous lesions and portable colposcopy has lower specificity, both methods of detection have low positive predictive value and high negative predictive value. In tandem, HPV testing and portable colposcopy had higher sensitivity for detection among women who underwent biopsies. In clinical practice, portable colposcopy was an effective, easy and affordable tool to transport to villages where cytology is not currently feasible.

\section{INTRODUCTION}

Over the past 50 years, cervical cancer morbidity and mortality has been greatly reduced due to earlier detection and treatment of pre-cancerous and cancerous lesions. However, cervical cancer remains among the most common gynecologic cancers in China. According to the 2015 China Cancer Statistics Data, the incidence of cervical cancer in 2015 was 98.9/10 000, with 11.3/10 000 originating from China's Southwest provinces, including Yunnan. ${ }^{1}$ Among women age 30-44 years, the incidence of cervical cancer was second only to breast cancer. ${ }^{1}$ Many women in more remote rural regions still lack any form of cervical cancer screening throughout their lifetime due to the economic and geographic challenges hindering access to care. Thus, cervical cancer screening in underserved regions of China remains a critical public health problem.

In the USA, the success of widespread screening over the past 60 years has reduced morbidity and mortality due to cervical cancer by $70 \%{ }^{2}$ In 2006 , the American Society for Colposcopy and Cervical Pathology published guidelines recommending a combined screening protocol with cytology and human papillomavirus (HPV) testing. The current US screening guidelines recommend cytology every 3 years for women age 21-29 years, and for women aged 30-65 screening every 3 years with cytology alone, every 5 years with high-risk HPV testing alone, or every 5 years with high-risk HPV testing in combination with cytology. ${ }^{3}$

In low and middle income countries, however, there is a dearth of effective, replicable screening programs; thus, many programs focus on finding alternative cost-effective approaches. Several largescale studies have demonstrated that HPV detection is superior to cytology for detection of high-grade cervical lesions. $^{4-7}$ In one large-scale randomized controlled trial in Italy, HPV-based screening was 


\section{Original Article}

found to be more effective than cytology in the prevention of invasive cervical cancer. This was attributed to better efficacy in early detection of clinically relevant lesions, treatment of cancers before invasion, and the ability to detect pre-cancerous changes that may not be clinically visible or even detectable with cytology. ${ }^{4}$ Furthermore, five trials with combined HPV and cytology testing versus HPV screening alone found that the overall combination was not more sensitive than sole HPV screening. ${ }^{5-9}$

While HPV has higher sensitivity than cytology and visual inspection with acetic acid, both visual inspection with acetic acid and cytology are more specific than HPV testing. However, it is also notable that the efficacy of cytologic examination and visual inspection with acetic acid may be influenced by the physician's skillset and may affect replicability of studying these methodologies.

For rural areas with limited medical resources, it is important to implement an appropriate cervical cancer screening program that takes into consideration local resource constraints in order to be feasible and sustainable. Portable colposcopy is a simplified version of traditional colposcopy, and it has the potential to improve the efficacy of visual screening methods. ${ }^{10}$ It is low-cost and easy to handle, which could help improve the feasibility. However, the validity of portable colposcopy requires further examination.

In this study we used a combination of HPV testing with portable colposcopy to compare the sensitivity and specificity of this combined screening method versus HPV testing alone. In February 2017, 488 women were screened in four townships of Yunnan Province.

\section{METHODS}

This study was conducted across four towns of Longyang District, Baoshan City, Yunnan Province, including Wafang, Yangliu, Yangyi and Xiyi, between February 12 and February 26, 2017. The protocol was approved by the Ethics Committees of The First People's Hospital of Yunnan and The Maternal and Child Health and Family Planning Service Center of Longyang District. Screening was incorporated with pre-existing screening, with HPV and subsequent thinprep cytology being offered by the Chinese government through a rural women's health screening program. This government-sponsored program is part of a larger rural women's health screening program which offers no-cost screening to women in remote villages.

Women were informed before the arrival of the medical team that cervical cancer screening would be taking place at the local clinics on a specified date and the government screening protocol mandated attendance for all women in the village. Two qualified gynecologists (HJ, BF) from The First People's Hospital of Yunnan and The Maternal and Child Health and Family Planning Service Center of Longyang District performed all screening examinations.

Women were excluded from the study if they had a known history of cervical intra-epithelial neoplasia or cervical cancer, hysterectomy with cervix removal, pre-existing conditions inhibiting safe examination (clotting or bleeding disorders), or the inability to provide informed consent.

All participants of this study underwent HPV testing and Gynocular (Gynius AB, Sweden) examination. The data were examined retrospectively to compare sensitivity, specificity, negative predictive value and positive predictive value of HPV and Gynocular testing versus HPV testing alone for the diagnosis of cervical cancer or pre-cancerous lesions.

Two qualified gynecologists performed the colposcopy examinations (HJ, BF) with the Gynocular colposcope. Photographs were taken with an iPhone 6 and an iPhone 6S. The examination operation was guided by China's Ministry of Health manual Technical Plan for Cervical Cancer Examination Project for Rural Women's Health.

Each woman was examined in a private clinic room. Subjects were asked to lie in a supine position for initial external inspection of the vulva and vagina. Using a portable speculum covered in plastic that was disposed of after each usage, the vagina and cervix were examined and HPV testing was then performed.

Cervical samples were obtained from women using a cytobrush for genomic DNA extraction. After HPV sampling was performed, the Gynocular portable colposcope was used to examine the cervix in the methodology typically performed with traditional colposcopy examination. First $3 \%$ acetic acid was applied for $30 \mathrm{~s}$ and observation was made lasting at least 2 min. Inspection was then performed after application of Lugol's $5 \%$ iodine.

If there was an obvious lesion by Gynocular examination, a biopsy was performed for histopathologic testing at the time of the examination unless the woman was currently menstruating or there was microscopic abnormal vaginal discharge suggesting infection. If the sample could not be obtained at the time of the examination, the participants were contacted for follow-up screening and treatment at The Maternal and Child Health and Family Planning Service Center of Longyang District 3 to 7 days after the cessation of menses or improvement of vaginal infection.

If the portable colposcopy testing was negative but HPV testing was subsequently found to be positive, women then had follow-up testing with thin-prep cytology and traditional colposcopy. Cervical biopsies were performed if there were any abnormalities. All histopathologic abnormalities were followed up with specific diagnosis and treatment, including loop electrosurgical excision procedure, cold knife conization or clinical follow up.

\section{HPV detection and histopathology}

HPV testing was performed by the PCR Laboratory of The National Health Commission's Key Laboratory, The First People's Hospital of Yunnan Province by liquid suspension chip technology with the Luminex 200 (Luminex Corp). Samples were stored at $4^{\circ} \mathrm{C}$ after collection and sent to The First People's Hospital of Yunnan for HPV genotyping. The Luminex-based HPV genotyping has been shown to be a highly sensitive, reproducible technique for simultaneous genotyping of genital HPV types. ${ }^{11}$ Target high-risk oncogenic types included: 16, 18, 31, 33, 35, 39, 45, 51, 52, 56, 58, 59, 66 and 68 . Thin-prep cytology and biopsies were interpreted by the Department of Pathology of the First People's Hospital of Yunnan Province.

The data were recorded and processed by Microsoft Excel, SPSS 13.0.

\section{RESULTS}

A total of 488 women aged 30 to 60 years (mean age $44.3 \pm 6.6$ years) were screened with combined HPV testing and portable colposcopy. The demographic details of the study participants are provided in Table 1. Among these women, 59.3\% had never 
Table 1 Characteristics of participants in the study

\begin{tabular}{|c|c|c|}
\hline Variables $(n=488)$ & $n$ & $\%$ \\
\hline \multicolumn{3}{|l|}{ Age (years) } \\
\hline $30-34$ & 43 & 8.8 \\
\hline 35-39 & 76 & 15.6 \\
\hline $40-44$ & 126 & 25.8 \\
\hline $45-49$ & 122 & 25.0 \\
\hline $50-54$ & 101 & 20.7 \\
\hline $55-60$ & 20 & 4.1 \\
\hline \multicolumn{3}{|l|}{ Education } \\
\hline None & 16 & 3.3 \\
\hline Primary & 292 & 59.8 \\
\hline Middle school & 158 & 32.4 \\
\hline High school & 16 & 3.3 \\
\hline Graduate & 6 & 1.2 \\
\hline \multicolumn{3}{|l|}{ Menopause } \\
\hline Post-menopausal & 77 & 15.8 \\
\hline Pre-menopausal & 403 & 82.6 \\
\hline Peri-menopausal & 8 & 1.6 \\
\hline \multicolumn{3}{|l|}{ Childbearing } \\
\hline Nil & 3 & 0.6 \\
\hline $1-3$ & 485 & 99.4 \\
\hline \multicolumn{3}{|l|}{$\begin{array}{l}\text { Lifetime number of } \\
\text { sexual partners }\end{array}$} \\
\hline One & 486 & 99.6 \\
\hline Two & 2 & 0.4 \\
\hline
\end{tabular}

received any prior cervical cancer screening, and only $16.2 \%$ reported having been screened within the past 3 years. A flow diagram is shown in Figure 1.
Table 2 Human papillomavirus (HPV) genotypes detected among study participants

\begin{tabular}{lrl}
\hline HPV genotype & $\mathbf{n}$ & $\%$ \\
\hline 16 & 4 & 0.8 \\
18 & 4 & 0.8 \\
31 & 1 & 0.2 \\
33 & 5 & 1.0 \\
35 & 2 & 0.4 \\
39 & 3 & 0.6 \\
45 & 0 & 0 \\
51 & 1 & 0.2 \\
52 & 15 & 3.1 \\
56 & 7 & 1.4 \\
58 & 6 & 1.2 \\
59 & 6 & 1.2 \\
66 & 1 & 0.2 \\
68 & 4 & 0.8 \\
\hline
\end{tabular}

Of the 488 women screened, $46(9.4 \%)$ tested positive for HPV. A single HPV type was detected in 34 women, while 12 women were co-infected with multiple genotypes (nine women with two genotypes, three women with three genotypes). The most common subtypes were HPV 52 and HPV 56. Results are shown in Table 2.

Among the 488 women screened with portable colposcopy, 24 women $(4.9 \%)$ underwent biopsy based on visual inspection with acetic acid-positive colposcopy screening. Of these women, 20 biopsies were obtained at the time of the initial colposcopy examination, while four were obtained later due to concern for infection or menstruation at the time of the initial examination. Of these 24 women, three were HPV positive and 21 were HPV negative.

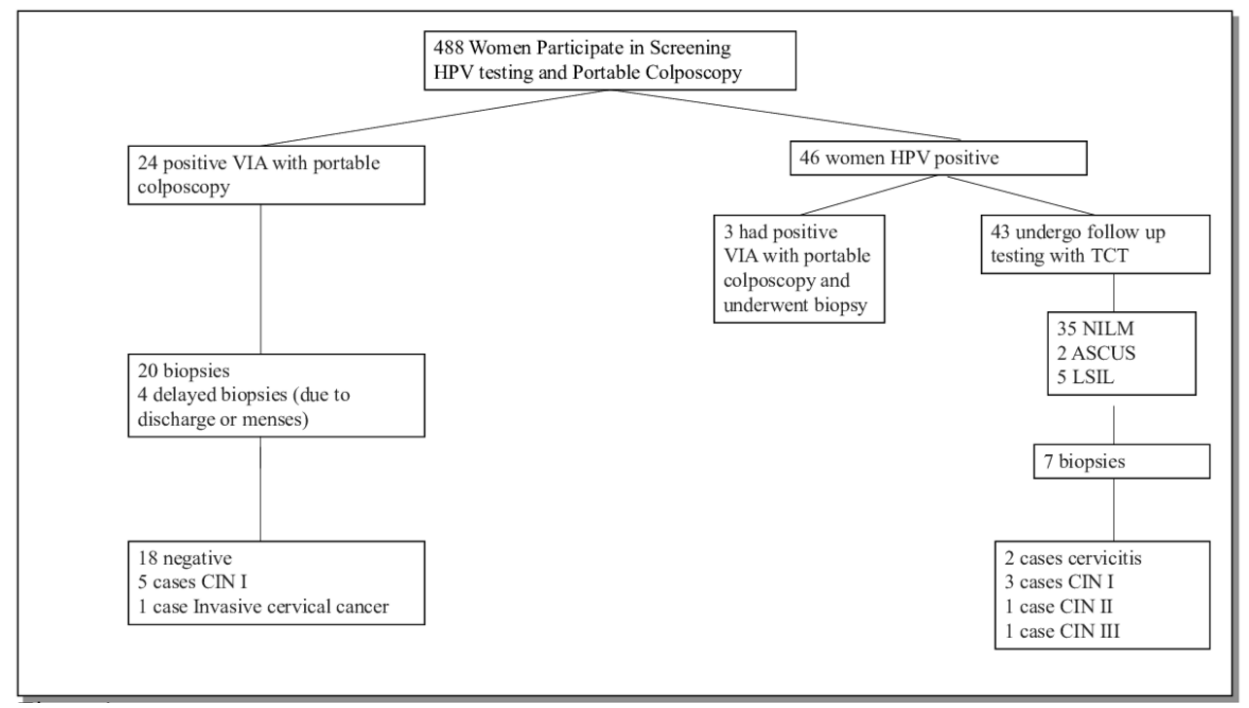

Figure 1 Flow diagram of participation and methodology of evaluation by human papillomavirus screening, portable colposcopy, thin-prep cytology and histologic biopsies. ASCUS, atypical squamous cells of undetermined significance; CIN, cervical intra-epithelial neoplasia; HPV, human papillomavirus; LSIL, low-grade squamous intra-epithelial lesions; NILM, negative for intra-epithelial lesion or malignancy; TCT, thin-prep cytology; VIA, visual inspection with acetic acid. 


\section{Original Article}

Table 3 Histopathology results from women screening positive with portable colposcopy

\begin{tabular}{ll}
\hline Negative (including cervicitis) & $\mathbf{1 8}$ \\
\hline CIN I & $\mathbf{5}$ \\
CIN II & 0 \\
CIN III & 0 \\
Invasive cervical cancer & $\mathbf{1}$ \\
\hline
\end{tabular}

$\mathrm{CIN}$, cervical intra-epithelial neoplasia.

Twenty-four women underwent cervical biopsies bases on positive screening by portable colposcopy examination. These results are reported in Table 3.

Forty-six women tested positive for HPV. One of these women had screened positive on preliminary colposcopy and her biopsy resulted in cervical intra-epithelial neoplasia (CIN) III and squamous cell carcinoma; she received treatment at The Maternal and Child Health and Family Planning Center. Another two women also had biopsies taken at the time of portable colposcopy and were diagnosed with CIN I and cervicitis. Finally, 43 women underwent follow-up testing with thin-prep cytology. Of these women, 36 were negative for intra-epithelial lesion or malignancy, two women had atypical squamous cells of unknown significance, and five had a low-grade squamous intraepithelial lesion.

All seven women with atypical squamous cells of unknown significance or low-grade squamous intra-epithelial lesion underwent biopsies. Their pathology results are shown in Table 4.

Women were categorized as disease positive if they were biopsied and the result demonstrated pre-cancerous or cancerous lesions, from CIN I through to squamous cell carcinoma. Women who were not biopsied because they had screened negative by portable colposcopy and were HPV negative or were HPV positive but subsequently had a negative thin-prep cytology result were considered true negatives for cancerous or pre-cancerous lesions.

The sensitivity, specificity, positive predictive value and negative predictive value of HPV screening alone, portable colposcopy alone, and combined screening with HPV and portable colposcopy for the detection of cancerous and pre-cancerous lesions (defined as CIN I through squamous cell carcinoma) are described in Table 5.

\section{DISCUSSION}

At present, cervical cancer screening in rural areas of Yunnan is supported by the local government by district with funding through

Table 4 Histopathology results from women screening positive with human papillomavirus testing and subsequent thin-prep cytology

\begin{tabular}{ll}
\hline Histopathology & Number \\
\hline Cervicitis & 2 \\
CINI & 3 \\
CIN II & 1 \\
CIN III & 1 \\
Cancer & 0 \\
\hline
\end{tabular}

CIN, cervical intra-epithelial neoplasia.
Table 5 Sensitivity, specificity, positive predictive value and negative predictive value of human papillomavirus screening, portable colposcopy, and combined screening method for detection of pre-cancerous and cancerous cervical lesions

\begin{tabular}{lcllc}
\hline Screening modality & $\begin{array}{l}\text { Sensitivity } \\
(\%)\end{array}$ & $\begin{array}{l}\text { Specificity } \\
(\%)\end{array}$ & $\begin{array}{l}\text { PPV } \\
\text { (\%) }\end{array}$ & $\begin{array}{l}\text { NPV } \\
(\%)\end{array}$ \\
\hline HPV testing alone & 63.6 & 91.8 & 15.2 & 99.1 \\
$\begin{array}{l}\text { Portable colposcopy } \\
\text { testing alone }\end{array}$ & 54.6 & 96.2 & 25.0 & 98.9 \\
$\begin{array}{l}\text { HPV testing and } \\
\text { portable colposcopy } \\
\text { combined }\end{array}$ & 100 & 88.3 & 16.4 & 100 \\
\hline
\end{tabular}

HPV, human papillomavirus; NPV, negative predictive value; PPV, positive predictive value.

the county level maternal and child health budget. There are many government-sponsored screening projects but no standardized screening methodology.

Thin-prep cytology sampling is feasible and quick to perform; however, many of the closest urban institutions do not have the technology or resources to carry out cytology or histopathology. Thus, they send these samples to other urban institutions at additional cost and follow up is challenging. Traditional colposcopy as performed in urban hospitals is not costly long-term, but the traditional scope is not easily transported outside of the clinic and it is not effective as a sole primary screening tool.

While HPVtesting has high sensitivity and specificity for the detection of pre-cancerous and cancerous lesions, and portable colposcopy has lower specificity, both methods of detection have low positive predictive value and high negative predictive value. In tandem, however, HPV testing with portable colposcopy had a much higher sensitivity for detection of pre-cancerous and cancerous lesions.

In clinical practice we also found that portable colposcopy was an easy and affordable tool to transport to the villages. The scopes are lightweight and have rechargeable batteries; therefore they do not require additional transport vehicles and manual support as was needed to carry a traditional colposcope. For these reasons, HPV testing combined with portable colposcopy may improve screening at a much lower cost, as the cost of colposcopy is significantly lower than thin-prep cytology. Furthermore, when an abnormal lesion is detected by portable colposcopy a biopsy can be performed immediately, which helps obviate the challenges of follow up.

The results of this study were based on examinations performed by a trained and well-experienced gynecologist; thus, our results are not transferable to communities without access to a trained colposcopist. Additionally, the gold standard for calculating sensitivity and specificity for cervical cancer screening is to have histopathology samples of cervical biopsies of all women who were screened (positive or negative). ${ }^{12}$ However, there were not sufficient resources to biopsy all women screened, and additionally there are risks associated with cervical biopsy. Thus, in this study not all women were biopsied. Women who were negative by portable colposcopy examination and HPV testing were considered disease-free; thus, it is possible that there is some overestimation of sensitivity and specificity. 
Throughout China HPV16 and HPV18 are widespread strains among women with cervical cancer; however, there is variability of genotypes by region. ${ }^{13}$ In one study on the prevalence of HPV infection among nearly 30000 Chinese women in Yunnan, the overall HPV prevalence was $12.9 \%$ with the three most prevalent high-risk HPV types being HPV52, HPV16 and HPV58. Age subgroup analysis also demonstrated bimodal peak prevalence: one among women under 25 , and another among women over 56 years. ${ }^{14}$

In this study the overall prevalence of HPV (9.4\%) was low when compared with rates throughout China and with a larger study in Yunnan (12.9\%). ${ }^{12}$ Additionally, it is notable that the most common types of HPV (HPV52, HPV 56, HPV58 and HPV59) did not mirror overall prevalence throughout the country as described in larger studies and meta-analyses.

Major risk factors for HPV infection include number of sexual partners, age at first intercourse, smoking, low socioeconomic status, and taking oral contraceptive pills. The low prevalence of HPV among the population of women screened in this study may be multifactorial. First, the age range we studied was 30-60 years, with three-quarters of women over the age of 40 . Thus, our study may have noted a higher prevalence had we also screened women under 25, given reports of bimodal distribution. Additionally, the majority of women in this study (99.6\%) all reported only ever having had one sexual partner, and use of oral contraception pills and smoking is generally low in this region, decreasing their risk factors for acquiring HPV. Low number of sexual partners is not unique to this study; in another cross-sectional study looking at HPV seropositivity in rural China, $99.9 \%$ of women reported 0-2 lifetime partners and only $0.1 \%$ reported $>3$ lifetime partners. ${ }^{15}$ However, our study was a small sample size and may not be representative of overall prevalence among the rural population of women in Yunnan.

Future studies should examine the sensitivity and specificity of portable colposcopy as compared with traditional colposcopy. More research is warranted to determine with greater statistical significance whether HPV combined with portable colposcopy could be scaled up to improve the bandwidth of screening in remote areas at a much lower cost. Recent studies in Africa have also looked at the use of a screen-and-treat approach with immediate thermocoagulation treatment after positive screening for HPV with visual inspection with acetic acid. ${ }^{12}$ Connecting naked eye visual inspection with acetic acid or portable colposcopy with cell phones may also provide remote consultation for guiding same day therapy. ${ }^{16}{ }^{17}$ Further research could examine the use of HPV testing in tandem with portable colposcopy and digital photography guiding same day treatment in rural China.

\footnotetext{
Author affiliations

${ }^{1}$ Department of Obstetrics and Gynecology, University of Massachusetts Medical School, Worcester, Massachusetts, USA

2Department of Pediatrics, The Children's Hospital of Philadelphia, Philadelphia, Pennsylvania, USA

${ }^{3}$ Kunming University of Science and Technology, Kunming, China

${ }^{4}$ Department of Obstetrics and Gynecology, National Health Commission's Key Laboratory for Healthy Births in Western China, First People's Hospital of Yunnan Province, Yunnan, China

${ }^{5}$ Maine Medical Partners Women's Health Division of Gynecologic Oncology, Portland, Maine, USA

${ }^{6}$ Maternal and Child Health and Family Planning Service Center of Longyang District, Yunnan, China
}

${ }^{7}$ Department of Microbiology and Physiological Systems, University of Massachusetts Medical School, Worcester, Massachusetts, USA

Funding Gynius AB, Stockholm, Sweden, donated the two Gynocular colposcopes to be used in the study. They did not have any role in preparing the study protocol, conducting the study, analysis or manuscript preparation. The study was supported by The First People's Hospital of Yunnan Province.

Patient consent Not required.

Provenance and peer review Not commissioned; externally peer reviewed.

Open access This is an open access article distributed in accordance with the Creative Commons Attribution Non Commercial (CC BY-NC 4.0) license, which permits others to distribute, remix, adapt, build upon this work non-commercially, and license their derivative works on different terms, provided the original work is properly cited, an indication of whether changes were made, and the use is non-commercial. See: http://creativecommons.org/licenses/by-nc/4.0

\section{REFERENCES}

1. Chen W, Zheng R, Baade PD, et al. Cancer statistics in China, 2015. CA Cancer J Clin 2016;66:115-32.

2. Feldman S. Making sense of the new cervical-cancer screening guidelines. N Engl J Med 2011;365:2145-7.

3. US Preventive Services Task Force, Curry SJ, Krist AH, et al. Screening for cervical cancer: US Preventive Services Task Force Recommendation Statement. JAMA 2018;320:674-86.

4. Castle PE, Stoler MH, Wright TC, et al. Performance of carcinogenic human papillomavirus (HPV) testing and HPV16 or HPV18 genotyping for cervical cancer screening of women aged 25 years and older: a subanalysis of the ATHENA study. Lancet Oncol 2011;12:880-90.

5. Monsonego J, Hudgens MG, Zerat L, et al. Evaluation of oncogenic human papillomavirus RNA and DNA tests with liquid-based cytology in primary cervical cancer screening: the FASE study. Int $J$ Cancer 2011;129:691-701.

6. Ronco G, Giorgi-Rossi P, Carozzi F, et al. Efficacy of human papillomavirus testing for the detection of invasive cervical cancers and cervical intraepithelial neoplasia: a randomised controlled trial. Lancet Oncol 2010;11:249-57.

7. Arbyn M, Ronco G, Meijer CJ, et al. Trials comparing cytology with human papillomavirus screening. Lancet Oncol 2009;10:935-6.

8. Zhao FH, Lin MJ, Chen F, et al. Performance of high-risk human papillomavirus DNA testing as a primary screen for cervical cancer: a pooled analysis of individual patient data from 17 population-based studies from China. Lancet Oncol 2010;11:1160-71.

9. Arbyn M, Sankaranarayanan R, Muwonge R, et al. Pooled analysis of the accuracy of five cervical cancer screening tests assessed in eleven studies in Africa and India. Int J Cancer 2008;123:153-60.

10. Basu P, Banerjee D, Mittal S, et al. Evaluation of a compact, rechargeable, magnifying device to triage VIA and HPV positive women in a cervical cancer screening program in rural India. Cancer Causes Control 2016;27:1253-9.

11. Oh Y, Bae SM, Kim YW, et al. Polymerase chain reaction-based fluorescent Luminex assay to detect the presence of human papillomavirus types. Cancer Sci 2007;98:549-54.

12. Chibwesha CJ, Frett B, Katundu K. Clinical performance validation of 4 point-of-care cervical cancer screening tests in HIV-infected women in Zambia. J Low Genit Tract Dis 2016;20:218-23.

13. Clifford GM, Rana RK, Franceschi S, et al. Human papillomavirus genotype distribution in low-grade cervical lesions: comparison by geographic region and with cervical cancer. Cancer Epidemiol Biomarkers Prev 2005;14:1157-64.

14. Li Z, Liu F, Cheng S, et al. Prevalence of HPV infection among 28,457 Chinese women in Yunnan Province, southwest China. Sci Rep 2016;6:21039.

15. Liu F, Deng Q, Zhang C, et al. Human papillomavirus DNA positivity and seropositivity in rural Chinese men and women: a population-based cross-sectional study. Sci Rep 2016;6:26343.

16. Bateman AC, Parham GP, Sahasrabuddhe VV, et al. Clinical performance of digital cervicography and cytology for cervical cancer screening in HIV-infected women in Lusaka, Zambia. J Acquir Immune Defic Syndr 2014;67:212-5.

17. DeGregorio G, Manga S, Kiyang E, et al. Implementing a Feefor-Service Cervical Cancer Screening and Treatment Program in Cameroon: Challenges and Opportunities. Oncologist 2017;22:850-9. 